\title{
Relativity of Electric Field to Resonance Characteristics and Piezoelectric Constants of Modified PZT Ceramics
}

\author{
Jin-Heon Oh, Jong-Nam Lim, Seung-su Lee, and Kee-Joe Lim* \\ Department of Electrical Engineering, Chungbuk National University, Cheongju 361-271, Republic of Korea
}

(Received August 13 2009, Revised October 12 2009, Accepted December 11 2009)

\begin{abstract}
The practical applications of piezoelectric ceramics are applied not only under low electric field environment. Therefore, an examination for characteristics of PZT ceramics under the high electric field condition can contribute to reducing the susceptibility of multifarious applications and to facilitating production of control circuits. These contributions can lead to the expansion of industrial applications. In this research, we fabricated disk-type PZT ceramic samples using conventional methods, measured the resonance characteristics of these samples under from low to high voltage conditions and calculated the PZT constants. [DOI: 10.4313/TEEM.2009.10.6.196]
\end{abstract}

Keywords: Electric field dependence, Non-linear characteristics, Material property, Modified PZT

\section{INTRODUCTION}

Since piezoelectric phenomenon able to develop an electric charge proportional to a mechanical stress discovered by J. and P. Curie first, this material has been utilized as core material in diversity fields [1]. Generally, material properties of PZT ceramics are measured under the small signal input. Concrete concept and process are stated clearly in the international standards [2]. The various material constants, determined by these methods are accepted as being trustworthy. In high power devices, such as ultrasonic motors and PZT actuators need a high level or great vibration amplitude, large input signals should be provided to the PZT elements. In accordance with many previous researches, material properties of PZT ceramics shows the linear characteristics only limited ranges of input voltages [3-5]. Hence, it is difficult to estimate the suitability of PZT ceramic material in case of high power applications. In Institute of Radio Engineers (IRE) standards, the material properties are able to be acquired from frequency-impedance characteristics of PZT ceramics [2]. Therefore, if these relations are varied with the applied input voltage, the material constants, calculated by the method enshrined in the standards, undergo changes necessarily. This fact signifies that the material properties of PZT ceramics are affected by the applied electric fields. In this study, we fabricated the disk type PZT ceramic specimens by conventional methods and considered the electric field dependence of frequencyimpedance characteristics. And also, we calculated the PZT constant $\mathrm{d}_{33}$ using the resonance method of the IRE standards from those frequencies representatively.

\section{EXPERIMENTS}

\subsection{Specimen fabrication}

We used PZT - PMNS ceramics as disk type specimens in this experiment. PZT-PMNS ceramics definite composition is shown as follows [6].

$$
0.9 \mathrm{~Pb}\left(\mathrm{Zr}_{0.51} \mathrm{Ti}_{0.49}\right) \mathrm{O}_{3}-0.1 \mathrm{~Pb}\left(\mathrm{Mn}_{1 / 3} \mathrm{Nb}_{1 / 3} \mathrm{Sb}_{1 / 3}\right) \mathrm{O}_{3}+0.05 \mathrm{Cr}_{2} \mathrm{O}_{3}
$$

\footnotetext{
* Author to whom corresponding should be addressed: electronic mail:kjlim@cbnu.ac.kr
}

To increase the dielectric and PZT properties of PZT ceramics significantly, $\mathrm{MnO}_{2}, \mathrm{Nb}_{2} \mathrm{O}_{5}, \mathrm{Sb}_{2} \mathrm{O}_{3}$ were added. Mixing ratio was determined by considering morphotropic phase boundary of ceramics. $\mathrm{Cr}_{2} \mathrm{O}_{3}$ was added to improve the mechanical quality factor $\left(\mathrm{Q}_{\mathrm{m}}\right)$ and to reduce the dielectric loss $(\tan \delta)$. This material shows excellent properties of high mechanical quality factor, high electromechanical coupling factor $\left(\mathrm{k}_{\mathrm{p}}\right)$ and low dielectric loss. The disk type ceramic specimens based on this material were fabricated. It had 28-mm diameter and 1-mm thickness.

\subsection{Measurement process}

The system for measuring resonance characteristics according to input voltage is presented in Fig. 1. Output signal of the built-in oscillator in the frequency response analyzer(FRA 5087; NF Corporation, Japan) was amplified by a power amplifier to drive the PZT specimen. The voltage was applied to the specimen and measured from $\mathrm{Ch} 1$ of FRA. The current flowing through the specimen was converted into voltage multiplied by the shunt resistor and was inputted through $\mathrm{Ch} 2$. By setting the analysis mode of the frequency response analyzer to $\mathrm{Ch} 1 / \mathrm{Ch} 2$, the impedance of the PZT specimen was measured. By measuring impedance by sweeping the frequency of the internal oscillator, the frequency characteristics of impedance were obtained. Resonance characteristics of the specimen of PZT ceramics are measured under the input voltage from $1 \mathrm{~V}_{\text {rms }}$ to $25 \mathrm{~V}_{\text {rms }}$.

\subsection{Calculation process of PZT constants $d_{33}$}

In order to analyze the variation of a PZT constant $d_{33}$ due to the change of the electric field, we calculated by means of the resonance method of the IRE standards. In accordance with the IRE standards, the value of $d_{33}$ can be reckoned by the following procedure step by step [2].

$$
k_{33}=\frac{\pi}{2} \frac{f_{r}}{f_{a}} \tan \left(\frac{\pi}{2} \cdot \frac{f_{a}-f_{r}}{f_{a}}\right)
$$

$$
\frac{1}{s_{33}^{D}}=4 \rho f_{a}^{2} l^{2}
$$




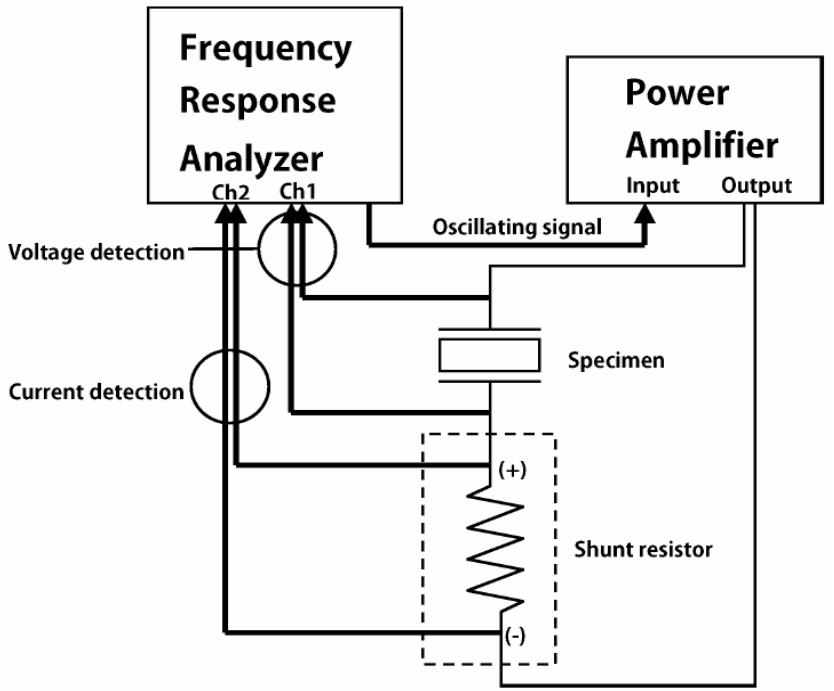

Fig. 1. Resonance characteristics measurement system.

$$
\begin{aligned}
& s_{33}^{E}=s_{33}^{D} /\left(1-k_{33}^{2}\right) \\
& d_{33}=k_{33} \sqrt{\epsilon_{33}^{T} s_{33}^{E}}
\end{aligned}
$$

The electro-mechanical coupling factor $\mathrm{k}_{33}$ is determined from the fundamental frequency $f_{a}$ and $f_{r}$ of thin cylinders. $F_{a}$ and $f_{r}$ mean the anti resonance frequency and resonance frequency respectively. $\mathrm{s}_{33}^{\mathrm{D}}$ is acquired by sample size, density and anti resonance frequency.

\section{RESULTS AND DISCUSSION}

\subsection{Frequency-impedance characteristics}

Impedance curves due to the magnitude of the input voltages are shown in Fig. 2. And the resonance and antiresonance frequencies in accordance with the voltage variation are shown in Table 1. Input voltage was from $1 \mathrm{~V}_{\mathrm{rms}}$ to $25 \mathrm{~V}_{\mathrm{rms}}$. In the first resonance mode of the specimen, the impedance according to the frequency was measured. When input voltages of $1 \mathrm{~V}_{\text {rms }}$ and $5 \mathrm{~V}_{\text {rms }}$ were applied, the impedance curve at the resonance and anti-resonance point had a bisymmetric shape and the value of impedance showed relatively large changes. But, when the input voltage was more than $10 \mathrm{~V}_{\mathrm{rms}}$, the impedance curve at the resonance point showed an asymmetric shape. Also, changes of the impedance value were relatively small. And this value varied steeply near the resonance frequency. Uchino et al. [7], explained that this phenomenon happened due to the non-linearity of the PZT characteristics using an electrical equivalent circuit. It seems that the variation of the frequency hardly harmonized with the mechanical vibration as the higher voltage was applied. As a result, the vibration change of dipoles which existed in the ceramic material seems to become sluggish.

Table 1. Resonance and anti-resonance frequencies in accordance with the voltage variation.

\begin{tabular}{ccccccc}
\hline & $1 \mathrm{~V}_{\text {rms }}$ & $5 \mathrm{~V}_{\text {rms }}$ & $10 \mathrm{~V}_{\text {rms }}$ & $15 \mathrm{~V}_{\text {rms }}$ & $20 \mathrm{~V}_{\mathrm{rms}}$ & $25 \mathrm{~V}_{\text {rms }}$ \\
\hline \hline $\mathrm{f}_{\mathrm{r}} \mathrm{kHz}$ & 83.024 & 82.976 & 81.683 & 81.491 & 81.149 & 80.761 \\
$\mathrm{f}_{\mathrm{a}} \mathrm{kHz}$ & 94.135 & 94.151 & 94.223 & 94.114 & 93.754 & 93.501 \\
\hline
\end{tabular}

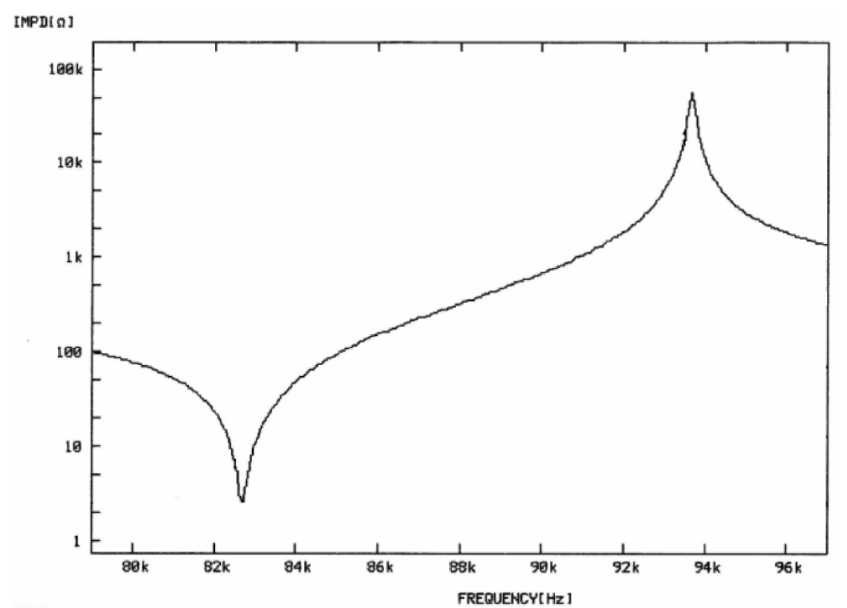

(a) $1 \mathrm{~V}_{\text {rms }}$

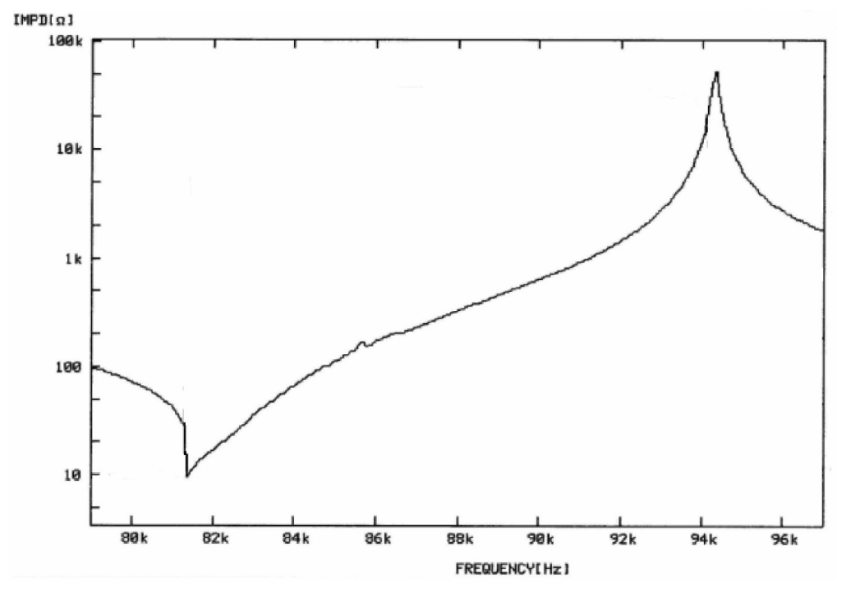

(b) $10 \mathrm{~V}_{\mathrm{rms}}$

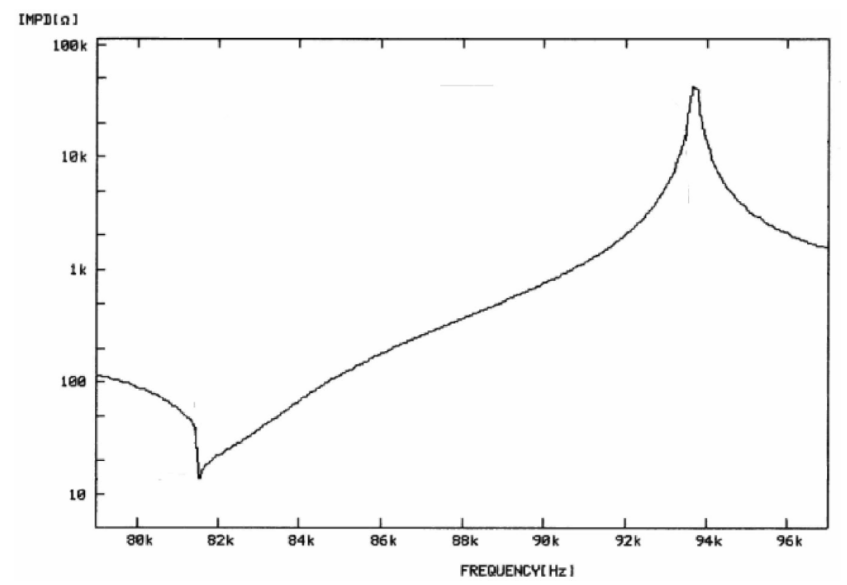

(c) $20 \mathrm{~V}_{\mathrm{rms}}$

Fig. 2. Impedance characteristics variation according to input voltage.

Table 2. Calculation values of $\mathrm{k}_{\mathrm{p}}$ and $\mathrm{Q}_{\mathrm{m}}$ of specimen.

\begin{tabular}{ccccccc}
\hline & $1 \mathrm{~V}_{\text {rms }}$ & $5 \mathrm{~V}_{\text {rms }}$ & $10 \mathrm{~V}_{\text {rms }}$ & $15 \mathrm{~V}_{\text {rms }}$ & $20 \mathrm{~V}_{\text {rms }}$ & $25 \mathrm{~V}_{\text {rms }}$ \\
\hline \hline $\mathrm{k}_{\mathrm{p}}$ & 0.4190 & 0.4201 & 0.4432 & 0.4447 & 0.4453 & 0.4480 \\
$\mathrm{Q}_{\mathrm{m}}$ & 158.72 & 157.80 & 140.63 & 139.70 & 139.79 & 138.42 \\
\hline
\end{tabular}

In IRE standards, the $\mathrm{k}_{\mathrm{p}}$ is obtained from the fundamental mode $f_{r}$ and $f_{a}$ of a thin disk as follows. 


$$
\begin{aligned}
& k_{p}^{2}=\frac{1}{p} \frac{f_{a}^{2}-f_{r}^{2}}{f_{a}^{2}} \\
& p=\frac{2\left(1+\sigma^{E}\right)}{\eta_{1}^{2}-1-\left(\sigma^{E}\right)^{2}}
\end{aligned}
$$

$\sigma^{\mathrm{E}}$ is Poisson's ratio, and $\eta_{1}$ is the lowest positive root of $\left(1+\sigma^{\mathrm{E}}\right) \mathrm{J}_{1}(\eta)=\eta J_{0}(\eta) . J_{0}$ is Bessel function of first kind and zero order, $J_{1}$ is Bessel function of first kind and first order [2].

The mechanical quality factor $\mathrm{Q}_{\mathrm{m}}$ is obtained from the determination of the minimum impedance $Z_{r}$ at the fundamental resonance. This measurement is accomplished by substitution of a variable resistance for the test specimen at $f_{r} . Q_{m}$ is given by the follow relation.

$$
\frac{1}{Q_{m}}=2 \pi f_{r} Z_{r} C^{F} \frac{f_{a}^{2}-f_{r}^{2}}{f_{a}^{2}} \approx 4 \pi Z_{r} C^{F}\left(f_{a}-f_{r}\right)
$$

$\mathrm{C}^{\mathrm{F}}$ is the capacitance measured under the low frequency condition $(1 \mathrm{kHz})$. Table 2 shows the calculation results of $\mathrm{k}_{\mathrm{p}}$ and $\mathrm{Q}_{\mathrm{m}}$ by the upper equations. In accordance with the input voltage variation, the electromechanical coupling coefficient $\mathrm{k}_{\mathrm{p}}$ is increased, but $\mathrm{Q}_{\mathrm{m}}$ is decreased slightly.

\subsection{Electric field - resonance frequencies variation}

Figure 3 represents the variation of resonance frequency, anti-resonance frequency and the voltage reduction ratio at the resonance frequency, respectively. Specimen in Fig. 3 is $28 \mathrm{~mm}$ diameter and $1-\mathrm{mm}$ thickness. When the electric field increased, the resonance frequency and the voltage reduction ratio decreased. The resonance characteristics were very similar tendencies as shown in the graphs. Peculiarity of these graphs was that the resonance frequency and voltage reduction ratio decrease steeply at a specific electric field, 5-10 $\mathrm{V}_{\mathrm{rms}} / \mathrm{mm}$ in Fig. 3. In case of the resonance frequencies in Fig. 3(a), the measured value at a 1 $\mathrm{V}_{\text {rms }} / \mathrm{mm}$ was $83.0 \mathrm{kHz}$. The measured value at $25 \mathrm{~V}_{\mathrm{rms}} / \mathrm{mm}$ was $80.8 \mathrm{kHz}$ Shift of the resonance frequency was about $2.2 \mathrm{kHz}$. As the electric field increased, the decrement of the resonance frequency was found. Therefore, the resonance frequency shift may have considerably occurred in the case of high power devices such as ultrasonic motors or micropumps, transformers etc. When the PZT ceramic resonated, the magnitude of the voltage across PZT ceramics decreased We compared the voltage drop with the actual input voltage and represented the voltage reduction ratio as shown below. This equation relates to the impedance drop characteristics of the specimens.

Voltage reduction ratio $(\%)=\frac{\text { Input voltage }- \text { voltage at the resonance frequency }}{\text { (n) }}$ Input voltage

Reduction ratio of the specimen of $28 \mathrm{~mm}$ in diameter shows about $91.7 \%$ under $1 \mathrm{~V}_{\mathrm{rms}} / \mathrm{mm}$ and $5.6 \%$ under 25 $\mathrm{V}_{\mathrm{rms}} / \mathrm{mm}$.

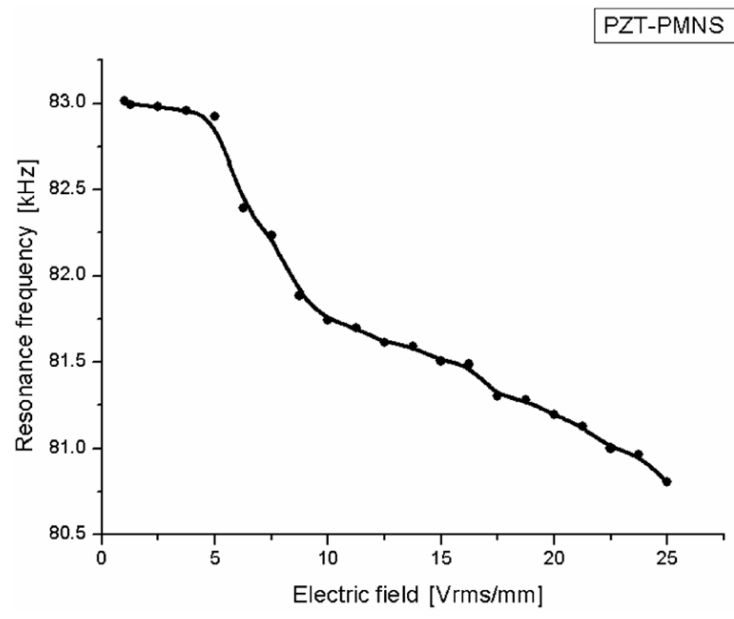

(a) Resonance frequency vs. electric field

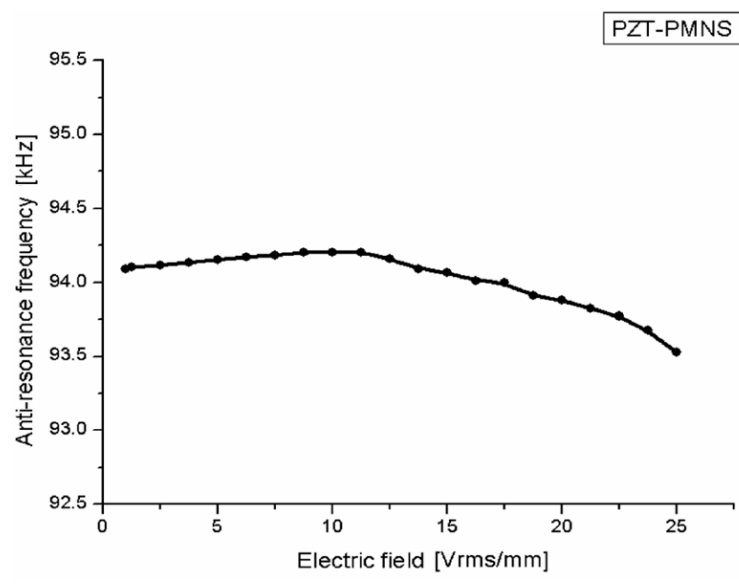

(b) Anti-resonance frequency vs. electric field

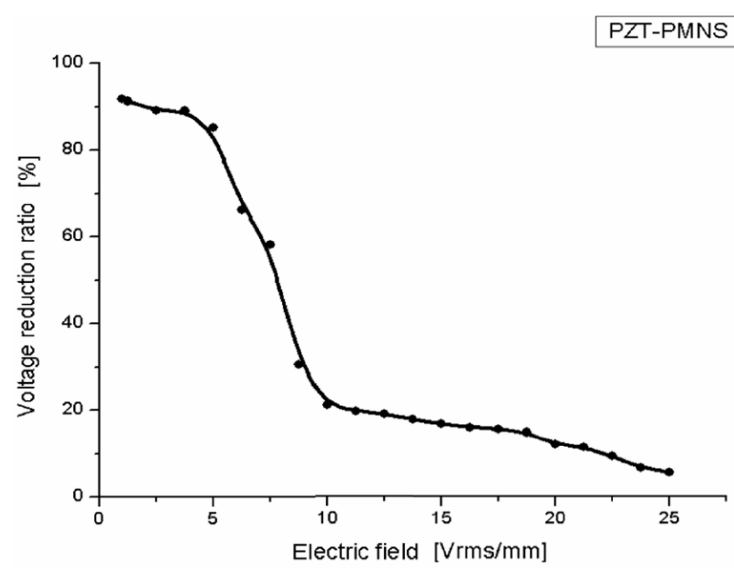

(c) Voltage reduction ratio vs. electric field

Fig. 3. Resonance characteristics of specimen. PZT: piezoelectric, PMNS: polynomial modular number system.

\subsection{Electric field - PZT constants $d_{33}$ variation}

Figure 4 shows variation of PZT constant $d_{33}$ for two cases. We calculated $d_{33}$ using the resonance method of the IRE standards [2]. Overall, as the electric field increased, the PZT constants increased. However, a non-linear characteristic was revealed in a portion of range of the magnitude of 


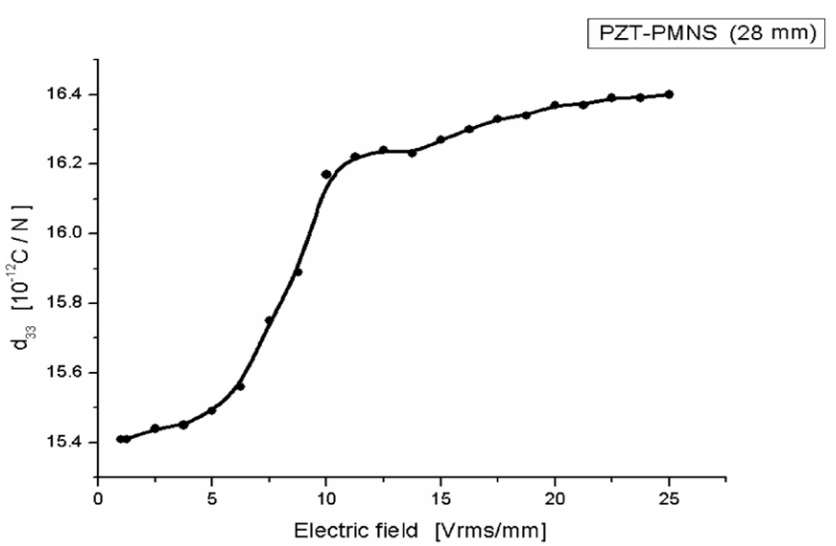

Fig. 4. Relation between PZT constant and electric field. PZT: piezoelectric, PMNS: polynomial modular number system.

the electric field. When the AC electric field, lower than the coercive field, was pushed in the polarized PZT ceramics, the domain of the grain changed and the strain of the PZT ceramics occurred. Hence, from the experimental results, the stacks and tendency which PZT constant increases shows that a difference exists in the shift of grain boundaries under low electric fields of nearly $1 \mathrm{~V}_{\mathrm{rms}} / \mathrm{mm}$ and over $10 \mathrm{~V}_{\mathrm{rms}} / \mathrm{mm}$. The observed increase in the $d_{33}$ is likely due to the larger extrinsic contribution resulting from increased domain switching under the larger electric fields [8].

\section{CONCLUSIONS}

Sherrit et al. [9] and Takahashi et al. [10] reported that mechanical factors had greater effects on the non-linearity than the electrical factors. In this experiment, an notable mechanical vibration factor, which exerted its influence on the PZT characteristics, was generated in the specified electrical realm. If the input voltage on the poled PZT ceramics increases, the vibration also axiomatically gets bigger at the resonance point. However, the vibration characteristics of PZT-PMNS ceramics are divided at the range of input voltages between $5 \mathrm{~V}_{\mathrm{rms}}$ and $10 \mathrm{~V}_{\mathrm{rms}}$. These could be ascertained by the non-linear change of the frequency, voltage drop and PZT constant $\mathrm{d}_{33}$. Because many applications such as PZT motor, pump and transformer etc. have a higher operational voltage level that the voltage used in this experiment, the variable tendencies of all of the above mentioned factors may be obvious.

\section{ACKNOWLEDGMENTS}

This work was supported by the Korea Research Foundation Grant funded by the Korean Government (KRF2008-313-D00376).

\section{REFERENCES}

[1] B. Jaffe, W. R. Cook, and H. L. Jaffe, Piezoelectric Ceramics (NonMetallic Solids, Vol. 3) (Academic Press, London, New York, 1971).

[2] Institute of Radio Engineers, Proc. IRE 49, 1161 (1961).

[3] R. S. Woollett and C. L. Leblanc, IEEE Trans. Son. Ultrason. 20, 24 (1973).

[4] J. H. Belding and M. G. McLaren, Am. Ceram. Soc. Bull. 49, 1025 (1970).

[5] S. Li, W. Cao, and L. E. Cross, J. Appl. Phys. 69, 7219 (1991).

[6] K. J. Lim, S. Y. Lee, J. S. Lee, M. J. Lee, and S. H. Kang, J. Electroceram. 13, 449 (2004).

[7] K. Uchino, H. Negishi, and T. Hirose, Jpn. J. Appl. Phys., Pt. 1, 28 (Suppl. 28-2), 47 (1989).

[8] A. J. Masys, W. Ren, G. Yang, and B. K. Mukherjee, J. Appl. Phys. 94, 1155 (2003).

[9] S. Sherrit, X. Bao, D. A. Sigel, M. J. Gradziel, S. A. Askins, B. P. Dolgin, and Y. Bar-Cohen, IEEE Ultrasonics Symposium (IEEE, Atlanta, GA, 2001) p. 1097.

[10] S. Takahashi, M. Yamamoto, and Y. Sasaki, Proceedings of the 11th IEEE International Symposium on Appliations of Ferroelectrics (ISAF-XI) (IEEE, Montreaux, Switz, 1998) p. 381. 